\title{
ОЦЕНКА РЕСУРСНО-ЗАТРАТНОГО РИСКА ПРИ ОРГАНИЗАЦИИ РЕМОНТНЫХ РАБОТ СЛОЖНОГО РОБОТИЗИРОВАННОГО ОБОРУДОВАНИЯ
}

\author{
Ю. М. Краковский ${ }^{\star}$, Н. А. Хоанг ${ }^{* *}$ \\ ${ }^{\star}$ Иркутский государственный университет путей сообщения \\ ${ }^{* *}$ Иркутский государственный аграрный университет им. А. А. Ежевского
}

Поступила в редакцию 28.06.2019 г.

\begin{abstract}
Аннотация. В данном исследовании обсуждается подход, предполагающий наличие страхового фонда, который выполняет две функции: накапливает платежи с различной периодичностью для выполнения различного вида ремонтных работ, а затем по мере необходимости оплачивает эти работы. Математическое описание организации ремонтных работ для роботизированного оборудования предлагается провести на основе случайного процесса риска, который описывает состояние страхового фонда. Этот подход позволил ввести понятие ресурсно-затратного риска, позволяющего количественно оценивать эффективность ремонтных работ с точки зрения их финансового обеспечения. Для его исследования создана моделирующая программа, основанная на событийном подходе. Эксперименты с помощью моделирующей программы позволили сделать вывод о том, что с точки зрения уменьшения ресурсно-затратного риска превышение дохода над расходами необходимо делать за счет начального годового значения страхового фонда. Некоторые выводы и утверждения являются логически очевидными, но анализ методом имитационного моделирования позволяет обосновать их количественно.
\end{abstract}

Ключевые слова: роботизированное оборудование, случайный процесс риска, ресурсно-затратный риск, имитационное моделирование.

\section{ВВЕДЕНИЕ}

Современное роботизированное оборудование является многокомпонентным, интегрирующим различные подсистемы: механическую, электрическую, электронную, информационно-управляющую и др. Это предъявляет дополнительные требования к его мониторингу и диагностике, а в дальнейшем к технологии и организации ремонтных работ.

Сложное многокомпонентное роботизированное оборудование в процессе эксплуатации функционирует в условиях неопределенности и требует различных технологий обслуживания: планово-предупредительных или по техническому состоянию. Последняя технология является предпочтительнее [1]. При этом необходимо учитывать, что ремонтные работы необходимо проводить при ограниченных финансовых и технологи-

(с Краковский Ю. М., Хоанг Н. А., 2019 ческих ресурсах. В связи с этим, поиск моделей для оценки эффективности управления ремонтными работами сложного оборудования является актуальной задачей, требующей своего решения.

В данном исследовании обсуждается подход, предполагающий наличие страхового фонда, который выполняет две функции: накапливает платежи с различной периодичностью для выполнения различного вида ремонтных работ, а затем по мере необходимости оплачивает эти работы. Этот подход позволяет ввести понятие ресурсно-затратного риска, основанного на случайном процессе риска. Важность методов оценки рисков в различных системах управления отмечается в опубликованных работах, например [2, 3].

Денежные потоки, связанные со страховым фондом, имеют следующую структуру:

1) платежи для различных видов ремонтных работ (накопление страхового фонда): а) текущие; б) аварийные; в) капитальные, 
связанные с модернизацией и развитием в процессе эксплуатации. Для каждого вида работ устанавливается периодичность пополнения страхового фонда (сутки) и их стоимость (млн. руб.);

2) затраты, необходимые для выполнения этих работ (уменьшение страхового фонда). Для каждого вида работ устанавливается периодичность использования страхового фонда (сутки) и их стоимость (млн. руб.).

Перейдем к математическому описанию организации ремонтных работ роботизированного оборудования на основе страхового фонда.

\section{МАТЕМАТИЧЕСКОЕ ОПИСАНИЕ ОРГАНИЗАЦИИ РЕМОНТНЫХ РАБОТ}

Математическое описание организации ремонтных работ для роботизированного оборудования предлагается провести на основе случайного процесса риска, который в нашем случае описывает состояние страхового фонда в момент времени $t-R(t)$. Возможность применения случайного процесса риска в качестве математической модели страхового фонда основана на применимости этого процесса при описании резерва страховой компании [4]. Использование случайного процесса риска при описании страхового фонда апробировано при исследовании различных видов работ для верхнего строения пути на железнодорожном транспорте $[5,6]$.

Для роботизированного оборудования первоначально формируется объем платежей для различных видов ремонтных работ на год ( $X$, млн.руб). Далее годовой объем платежей распределяется по видам работ

$$
\begin{aligned}
& X^{(1)}=c_{1} \cdot X, X^{(2)}=c_{2} \cdot X, \\
& X^{(3)}=c_{3} \cdot X ; c_{1}+c_{2}+c_{3}=1 .
\end{aligned}
$$

Здесь $c_{1}$ - коэффициент, учитывающий долю объема платежей для выполнения текущих ремонтных работ, $X^{(1)}$ - годовые платежи в страховой фонд для выполнения текущих ремонтных работ; $c_{2}$ - коэффициент, учитывающий долю объема платежей для выполнения аварийных ремонтных работ, $X^{(2)}-$ годовые платежи в страховой фонд для вы- полнения аварийных ремонтных работ; $c_{3}-$ коэффициент, учитывающий долю объема платежей для выполнения капитальных ремонтных работ, $X^{(3)}$ - годовые платежи в страховой фонд для выполнения капитальных ремонтных работ.

Стоимость одного платежа в страховой фонд для $j$-го вида ремонтных работ с учетом (1)

$$
\begin{gathered}
Y_{j}=h_{j} \cdot X^{(j)} / T g=c_{j} \cdot h_{j} \cdot X / T g, \\
j=1,2,3,
\end{gathered}
$$

где $T g$ - число суток в году; $h_{j}$ - периодичность платежей в страховой фонд (сутки) для $j$-го вида работ. В данном исследовании эти интервалы времени являются детерминированными величинами.

Суммарные накопления платежей в страховой фонд для выполнения ремонтных работ $j$-го вида с учетом (1) и (2) и сделанных предположений о периодичности его пополнения равны

$$
\begin{aligned}
Y_{j}(t)=Y_{j} \cdot N_{j}(t) & =\left(c_{j} \cdot h_{j} \cdot X / T g\right) \cdot N_{j}(t), \\
j & =1,2,3,
\end{aligned}
$$

где $N_{j}(t)$ - число платежей в страховой фонд за время $t$ для $j$-го вида работ.

Время свершения аварийных ситуаций описывается процессом

$$
T_{i}=T_{i-1}+t_{i}, i=1,2 \ldots, N_{a}(t), T_{0}=0,
$$

где $t_{i}$ - интервалы времени между аварийными ситуациями; $N_{a}(t)$ - число аварийных ситуаций за время $t$. В условиях неопределенности интервалы $t_{i}$ являются значениями случайной величины с заданной функцией распределения. Используя рекомендации литературных источников по теории надежности и диагностике [7, 8], можно в качестве законов распределения для времени наработки порекомендовать нормальное, Вейбулла, гамма, Бирнбаума-Саундерса и другие распределения.

Времени $T_{i}(4)$ соответствуют затраты на ликвидацию $i$-й аварийной ситуации $-Z_{i}$. Эти затраты являются случайной величиной с известной функцией распределения. Учитывая рекомендации по математике рискового страхования [9], в качестве закона распределения для затрат по ликвидации аварийной 
Оиенка ресурсно-затратного риска при организации ремонтных работ сложного роботизированного...

ситуации предложено использовать распределение Парето с нулевой точкой. Для этой случая функция распределения имеет вид

$$
F(z)=1-1 /(1+z / \beta)^{\alpha}, \quad z>0,
$$

а числовые характеристики (математическое ожидание, дисперсия и коэффициент вариации) равны

$$
\begin{gathered}
M=\beta /(\alpha-1), \\
D_{z}=\alpha \cdot \beta^{2} /\left[(\alpha-1)^{2} \cdot(\alpha-2)\right], \quad \alpha>2 ; \\
k v=\sqrt{\alpha /(\alpha-2)}, \quad k v>1 .
\end{gathered}
$$

Для распределения Парето с нулевой точкой параметры методом моментов с учетом (6) и (7) равны

$$
\begin{aligned}
& \alpha=2 \cdot k v^{2} /\left(k v^{2}-1\right) ; \quad k v>1 ; \\
& \beta=(\alpha-1) \cdot M .
\end{aligned}
$$

С учетом (5), моделирование значений случайной величины для распределения Парето с нулевой точкой осуществляется по формуле

$$
z=\beta \cdot\left[1 /(1-\mathrm{r})^{1 / \alpha}-1\right], \alpha>2,
$$

где $r$ - значение псевдослучайной величины, равномерно распределенной на интервале $(0,1)$. Учитывая (9), суммарные затраты на ремонтные работы по аварийным ситуациям за время $t$ равны

$$
Y A(t)=\sum_{i=1}^{N a(t)} z_{i}
$$

Для периодичности текущих работ также предполагается наличие неопределенности, что позволяет считать интервалы между этими работами случайной величиной (при необходимости эти интервалы можно считать детерминированными величинами). В связи с этим, время начала $i$-й текущей работы равно

$$
G_{i}=G_{i-1}+g_{i}, i=1,2 \ldots, N_{T}(t), G_{0}=0,
$$

где $g_{i}$ - интервалы времени между текущими работами; $N_{T}(t)$ - число текущих работ за время $t$. Как уже отмечалось выше, эти интервалы являются либо детерминированными, либо значениями случайной величины с известной функцией распределения.

Времени $G_{i}$ (11) соответствуют затраты на выполнение $i$-й текущей работы - $U_{i}$. Эти затраты являются случайной величиной с известной функцией распределения. В качестве вероятностных моделей для этих затрат рекомендуются нормальное или гамма распределения. Возможны и другие распределения.

С учетом сделанных предположений суммарные затраты на текущие работы за время $t$ равны

$$
Y T(t)=\sum_{i=1}^{N_{T}(t)} u_{i}
$$

где $u_{i}$ - значение затрат по $i$-й текущей работе.

Аналогично можно найти суммарные затраты на выполнение капитальных ремонтных работ

$$
Y K(t)=\sum_{i=1}^{N_{k}(t)} \omega_{i},
$$

где $\omega_{i}$ - значение затрат по $i$-й капитальной работе; $N_{k}(t)$ - число капитальных работ за время $t$. Интервалы времени и сами затраты являются либо детерминированными, либо случайными величинами с известными законами распределения.

В этих обозначениях состояние страхового фонда описывается случайным процессом риска

$$
\begin{aligned}
R(t)= & X_{0}+Y 1(t)+Y 2(t)+Y 3(t)- \\
& -Y A(t)-Y T(t)-Y K(t),
\end{aligned}
$$

где $X_{0}$ - начальные средства страхового фонда; $Y j(t)$ - суммарные накопления платежей по видам работ (3) к моменту времени $t$, $(j=1,2,3) ; \quad Y A(t)$ - суммарные затраты по аварийным ситуациям (10); $Y T(t)$ - суммарные затраты для выполнения текущих работ $(12) ; Y K(t)$ - суммарные затраты для выполнения капитальных работ (13).

Для исследования случайного процесса риска (14) предлагается использовать метод имитационного моделирования. Модели моделирования значений случайных величин приведены в специальной литературе, например $[10,11]$.

\section{МОДЕЛИ ВЫЧИСЛЕНИЯ РЕСУРСНО-3АТРАТНОГО РИСКА}

Для случайного процесса риска (14) определяется момент времени $\tau$, когда первый раз выполняется условие $R(t)<0$,

$$
\tau=\min _{t}\{t: \mathrm{R}(\mathrm{t})<0\} .
$$


Момент времени (15) характеризует эффективность организации ремонтных работ с точки зрения распределения платежей по их видам (1). Этот момент времени предлагается рассматривать как ресурсо-затратный риск и оценивать его показателем $r_{\tau}$, как вероятность специального события

$$
r_{\tau}=P\left(\tau<T_{\tau}\right)
$$

где $T_{\tau}$ - заданное время (сутки).

Риск (15) назван ресурсо-затратным, так как он оценивает модель «Ресурсы-затраты» для ремонтных работ, связанных с процессом эксплуатации роботизированного сложного оборудования. Ресурсы - это платежи для выполнения ремонтных работ в страховой фонд; затраты - это оплата ремонтных работ различных видов. Разность между ресурсами (доходами) и затратами должна быть положительной, иначе возникает риск невыполнения этих работ.

Величина (15) является случайной и несобственной т. к. для процесса (14) не всегда выполняется условие (15) [4]. В связи с этим вероятности

$$
P(\tau<\infty)<1, P\left(\tau>T_{i}\right)>0 .
$$

Здесь $T_{м}$ - максимальное время создания реализации процесса (14) при имитационном моделировании, $T_{\tau} \leq T_{м}$. При использовании имитационного моделирования для показателя (15), когда

$$
0<\tau \leq T_{M},
$$

создается выборка, которая в дальнейшем обрабатывается.

В данном исследовании при обработке этой выборки создаются точечная $\left(\tilde{R}_{\tau}\right)$ и интервальная $\left(\tau_{1}, \tau_{2}\right)$ оценки для показателя ресурсо-затратного риска (16) при различных значениях $T_{\tau}$.

Точечная оценка

$$
\tilde{R}_{\tau}=k_{\tau} / n,
$$

где $k_{\tau}$ - число реализаций процесса (14) для которых выполняется условие $\tau<T_{\tau}, \quad n-$ число созданных реализаций методом имитационного моделирования.

Интервальная оценка [12]

$$
\tau_{1}=k_{\tau} /\left[k_{\tau}+\left(n-k_{\tau}+1\right) \cdot F_{1}\left(v_{1}, v_{2}\right)\right],
$$

где $F_{1}\left(v_{1}, v_{2}\right)$ - критическое значение для
$F$-распределения при $v_{1}$ и $v_{2}$ степенях свободы и доверительной вероятности $\gamma=0,95$; $v_{1}=2 \cdot\left(n-k_{\tau}+1\right), \quad v_{2}=2 \cdot k_{\tau}$;

$$
\tau_{2}=\frac{\left(k_{\tau}+1\right) \cdot F_{2}\left(v_{3}, v_{4}\right)}{\left[n-k_{\tau}+\left(k_{\tau}+1\right) \cdot F_{2}\left(v_{3}, v_{4}\right)\right]},
$$

где $F_{2}\left(v_{3}, v_{4}\right)$ - критическое значение для $F$-распределения при $v_{3}$ и $v_{4}$ степенях свободы и доверительной вероятности $\gamma=0,95$; $v_{3}=2 \cdot\left(k_{\tau}+1\right), v_{4}=2 \cdot\left(n-k_{\tau}\right)$.

\section{ИССЛЕДОВАНИЕ \\ РЕСУРСНО-ЗАТРАТНОГО РИСКА МЕТОДОМ ИМИТАЦИОННОГО МОДЕЛИРОВАНИЯ}

Для исследования показателя (16) создана моделирующая программа, которая создает реализации случайного процесса риска (14), формирует выборку величины (15) и вычисляет точечную (19) и интервальную (20), (21) оценки показателя ресурсо-затратного риска.

Исследование проведено при следующих исходных данных:

1) годовой объем платежей $X=284,0$ млн. руб.;

2) число суток в году $T g=360$ сут.;

3) периодичность платежей для выполнения текущих ремонтных работ $h_{1}=10$ сут. (один платеж в начале каждой декады месяца);

4) периодичность платежей для выполнения аварийных ремонтных работ $h_{2}=30$ сут. (один платеж в начале каждого месяца);

5) периодичность платежей для выполнения капитальных ремонтных работ $h_{3}=90$ сут. (один платеж в начале каждого квартала).

6) доля объема платежей для выполнения текущих ремонтных работ $c_{1}=0,296$;

7) доля объема платежей для выполнения аварийных ремонтных работ $c_{2}=0,282$;

8) доля объема платежей для выполнения капитальных ремонтных работ $c_{3}=0,422$;

9) число моделируемых реализаций процесса (14) $n=20000$;

10) максимальное время моделирования реализации процесса (14) $T_{м}=1800$ сут.

Размер платежей (2) при этих исходных данных: $Y_{1}=2,334$ млн. руб., $Y_{2}=6,667$ млн. руб., $Y_{3}=30,0$ млн.руб. 
Оиенка ресурсно-затратного риска при организации ремонтных работ сложного роботизированного...

Моделирующая программа, используя событийный подход, воспроизводит три процесса поступления платежей по видам работ и три процесса по затратам на эти работы, накапливая величины (3), (10), (12), (13).

В табл. 1 приведены законы распределения и их числовые характеристики для интервалов времени между видами работ и затратам на эти работы: $m$ - математическое ожидание, $k_{v}$ - коэффициент вариации. Выбор законов распределения и их числовых характеристик предлагается осуществлять с помощью экспертов.

При выбранных исходных данных величина поступления платежей (доходы) и величина расходов на ремонты в среднем равны между собой. В страховой математике время (15) называют моментом разорения. Вероятность разорения будет меньше 1, если доходы будут превышать расходы. Это можно сделать различными способами, мы рассмотрели три варианта:

1) Вариант $A$, когда превышение дохода осуществляется за счет ежегодного начального значения страхового фонда $X_{0}$. Предлагается рассмотреть три случая, когда это значение равно 5, 10 и 20 процентов относительно годового объема платежей $X=284,0$ млн.руб.:

a) $X_{0}=14,2$ млн.руб.; b) $X_{0}=28,4$ млн. руб.; с) $X_{0}=56,8$ млн.руб.;

2) Вариант $B$, когда превышение дохода осуществляется за счет увеличения годового объема платежей $X, X_{0}=0$. В этом варианте также предлагается рассмотреть три случая, когда увеличение равно 5, 10 и 20 процентов: a) $X=298,2$ млн.руб.; b) $X=312,4$ млн.руб.; c) $X=340,8$ млн.руб;
3) Вариант $C$, когда превышение дохода осуществляется за счет полугодового начального значения страхового фонда $X_{0}$. Предлагается рассмотреть три случая, когда это значение за год равно 5, 10 и 20 процентов относительно годового объема платежей $X=284,0$ млн. руб. Полугодовые значения равны: а) $X_{0}=7,1$ млн. руб.; b) $X_{0}=14,2$ млн. руб.; с) $X_{0}=28,4$ млн. руб.

Подчеркнем, что суммарные годовые платежи во всех трех вариантах одинаковые.

Рассмотренные варианты предлагается промоделировать для четырех значений величины $T_{\tau}: 30$ сут., 90 сут., 180 сут., 360 сут.

В табл. 2 приведены результаты расчетов точечной и интервальной оценок показателя ресурсо-затратного риска (16): $P_{1}$ и $P_{2}$ это варианты исследования. Всего проведено 36 различных просчетов моделирующей программы.

Анализ значений табл. 2 позволяет сделать однозначный вывод о том, что с точки зрения уменьшения ресурсно-затратного риска превышение дохода над расходами необходимо делать по варианту $A$, когда это превышение осуществляется за счет начального годового значения страхового фонда $X_{0}$. Уверенность этого вывода обосновывается тем, что отличия точечных оценок подтверждается не пересечением доверительных интервалов ресурсно-затратного риска. Этот факт позволяет выбрать статистическую гипотезы о том, что показатель ресурсо-затратного риска для варианта $A$ меньше, чем для вариантов $B$ и $C$.

Варианты $A$ и $C$ статистически одинаковы лишь при $T_{\tau}=30$ сут.; вариант $C$ предпочтительнее, чем вариант $B$.

Таблица 1

Законы распределения и их числовые характеристики

\begin{tabular}{|l|c|c|c|c|}
\hline \multicolumn{1}{|c|}{ Работы } & \multicolumn{2}{|c|}{ Интервалы времени, сут. } & \multicolumn{2}{c|}{ Затраты, млн.руб. } \\
\hline \multirow{2}{*}{ Текущие } & \multicolumn{2}{|c|}{ Бирнбаума-Саундерса } & \multicolumn{2}{c|}{ Логнормальное } \\
\cline { 2 - 5 } & $m=15,0$ & $k_{v}=0,20$ & $m=3,5$ & $k_{v}=0,20$ \\
\hline \multirow{2}{*}{ Аварийные } & \multicolumn{2}{|c|}{ Вейбулла } & \multicolumn{2}{c|}{ Парето } \\
\cline { 2 - 5 } & $m=45,0$ & $k_{v}=0,30$ & \multicolumn{2}{c|}{ Нормальное } \\
\hline \multirow{2}{*}{ Капитальные } & \multicolumn{2}{|c|}{ Гамма } & $m=20,0$ & $k_{v}=0,15$ \\
\cline { 2 - 5 } & $m=60,0$ & $k_{v}=0,25$ & 20 \\
\hline
\end{tabular}


Таблица 2

Результаты расчетов точечной и интервальной оченок показателя ресурсо-затратного риска

\begin{tabular}{|c|c|c|c|c|c|c|c|c|c|c|}
\hline \multirow{4}{*}{$P_{2}$} & $P_{1}$ & \multicolumn{4}{|c|}{$a$} & \multicolumn{3}{c|}{$b$} & \multicolumn{3}{c|}{$c$} \\
\cline { 2 - 12 } & $T_{\tau}$ & $\tilde{R}_{\tau}$ & $\tau_{1}$ & $\tau_{2}$ & $\tilde{R}_{\tau}$ & $\tau_{1}$ & $\tau_{2}$ & $\tilde{R}_{\tau}$ & $\tau_{1}$ & $\tau_{2}$ \\
\hline \multirow{4}{*}{$A$} & 30 & 0,003 & 0,003 & 0,004 & 0,001 & 0,001 & 0,002 & 0,001 & 0,0 & 0,001 \\
\cline { 2 - 12 } & 90 & 0,068 & 0,065 & 0,071 & 0,036 & 0,034 & 0,039 & 0,012 & 0,010 & 0,013 \\
\cline { 2 - 12 } & 180 & 0,152 & 0,148 & 0,157 & 0,088 & 0,085 & 0,092 & 0,031 & 0,029 & 0,033 \\
\cline { 2 - 12 } & 360 & 0,278 & 0,273 & 0,284 & 0,182 & 0,178 & 0,185 & 0,078 & 0,075 & 0,081 \\
\hline \multirow{4}{*}{$B$} & 30 & 0,006 & 0,005 & 0,007 & 0,005 & 0,004 & 0,006 & 0,004 & 0,003 & 0,005 \\
\cline { 2 - 12 } & 90 & 0,130 & 0,126 & 0,133 & 0,109 & 0,105 & 0,112 & 0,075 & 0,072 & 0,078 \\
\cline { 2 - 11 } & 180 & 0,230 & 0,225 & 0,235 & 0,184 & 0,179 & 0,188 & 0,119 & 0,115 & 0,123 \\
\cline { 2 - 11 } & 360 & 0,336 & 0,331 & 0,342 & 0,264 & 0,259 & 0,269 & 0,157 & 0,153 & 0,161 \\
\hline \multirow{4}{*}{$C$} & 30 & 0,004 & 0,003 & 0,005 & 0,003 & 0,002 & 0,004 & 0,001 & 0,001 & 0,002 \\
\cline { 2 - 11 } & 90 & 0,107 & 0,103 & 0,111 & 0,068 & 0,065 & 0,071 & 0,031 & 0,029 & 0,033 \\
\cline { 2 - 11 } & 180 & 0,212 & 0,207 & 0,217 & 0,153 & 0,149 & 0,157 & 0,083 & 0,080 & 0,087 \\
\cline { 2 - 10 } & 360 & 0,312 & 0,307 & 0,318 & 0,223 & 0,218 & 0,228 & 0,115 & 0,112 & 0,119 \\
\hline
\end{tabular}

Дополнительно можно утверждать, что с увеличением начального значения ресурсно-затратный риск уменьшается, а с увеличением времени $T_{\tau}$ увеличивается. Последние утверждения являются логически очевидными, но анализ методом имитационного моделирования позволяет количественно оценить эти изменения.

\section{ЗАКЛЮЧЕНИЕ}

1. В работе предложен и реализован подход, предполагающий наличие страхового фонда, который выполняет две функции: накапливает платежи с различной периодичностью для выполнения различного вида ремонтных работ, а затем по мере необходимости оплачивает эти работы. Математическое описание организации ремонтных работ для роботизированного оборудования предлагается провести на основе случайного процесса риска, который описывает состояние страхового фонда.

2. Этот подход позволил ввести понятие ресурсно-затратного риска, позволяющего количественно оценивать эффективность ремонтных работ с точки зрения их финансового обеспечения. Для его исследования создана моделирующая программа, основанная на событийном подходе.
3. Эксперименты с помощью моделирующей программы позволили сделать вывод о том, что с точки зрения уменьшения ресурсно-затратного риска превышение дохода над расходами необходимо делать за счет начального годового значения страхового фонда. Уверенность этого вывода обосновывается тем, что отличия точечных оценок подтверждается не пересечением доверительных интервалов ресурсно-затратного риска. Некоторые выводы и утверждения являются логически очевидными, но анализ методом имитационного моделирования позволяет обосновать их количественно.

\section{СПИСОК ЛИТЕРАТУРЫ}

1. Ящура, А. И. Система технического обслуживания и ремонта общепромышленного оборудования: справочник / А. И. Ящура. М. : ЭНАС, 2012. - 360 c.

2. Браун А. А. Методы оценки рисков в системе управления безопасностью труда / А. А. Браун // Gaudeamus Igitur. - 2015. - № 4.

3. Вишняков, Я. Д. Общая теория рисков / Я. Д. Вишняков, Н. Н. Радаев. - М. : Изд-во «Академия», 2007. - 368 с.

4. Королев, В. Ю. Математические основы теории рисков. / В. Ю. Королев, В. Е. Бенинг, 
Оиенка ресурсно-затратного риска при организации ремонтных работ сложного роботизированного...

С. Я. Шоргин - М. : ФИЗМАТЛИТ, 2011. $620 \mathrm{c}$.

5. Начигин, В. А. Формализация селективной технологии содержания инфраструктуры и страховой фонд / Ю. М. Краковский, В. А. Начигин // Мир транспорта. - 2015. - Т. 13. - № 1. - С. 94-99.

6. Краковский, Ю. М. Моделирование перевозочного процесса железнодорожным транспортом: анализ, прогнозирование, риски / Ю. М. Краковский, С. К. Каргапольцев, В. А. Начигин; под ред. проф. Ю. М. Краковского. - СПб. : «ЛИТЕО», 2018. - 240 с.

7. Байхельт, Ф. Надежность и техническое обслуживание. Математический подход / Ф. Байхельт, П. Франкен. - М. : Радио и связь, 1988. - 392 с.

8. Краковский Ю. М. Математические и программные средства оценки техническо- го состояния оборудования / Ю. М. Краковский. - Новосибирск : Наука, 2006. - 228 с.

9. Мак Томас. Математика рискового страхования / Томас М. Пер. с нем. - М. : ЗАО «Олимп-Бизнес», 2005. - 432 с.

10. Кельтон, В. Имитационное моделирование / В. Кельтон, А. Лоу. - СПб. : Питер, 2004. - 847 c.

11. Краковский, Ю. М. Математическое обеспечение по моделированию случайных величин при вероятностном анализе безубыточности / Ю. М. Краковский, С. Г. Калиновский, А. С. Селиванов // Информационные технологии и проблемы математического моделирования сложных систем. - 2009. - № 7. С. $105-111$.

12. Закс, Л. Статистическое оценивание / Л. Закс. - М. : Статистика, 1976. - 598 с.

Краковский Юрий Мечеславович - д-р техн. наук, профессор, профессор кафедры «Информационные системы и защита информации», Иркутский государственный универси-тет путей сообщения. E-mail: 79149267772@yandex.ru

Хоанг Нгок Ань - аспирант кафедры информатики и математического моделирования, Иркутский государственный аграрный университет имени А. А. Ежевского.

E-mail: hoangngocanhmta@gmail.com

\title{
ASSESSMENT OF THE RESOURCE-COST RISK WHEN ORGANIZING REPAIR WORKS OF COMPLEX ROBOTIC EQUIPMENT
}

\author{
Y. M. Krakovsky , N. A. Hoang ${ }^{\star *}$ \\ ${ }^{*}$ Irkutsk State Transport University \\ ${ }^{*}$ The Irkutsk State Agrarian University named after A. A. Ezhevsky
}

\begin{abstract}
Annotation. This study discusses an approach that assumes the existence of an insurance fund that performs two functions: it accumulates payments at various intervals to perform various types of repair work, and then it pays for these works as needed. A mathematical description of the organization of repair work for robotic equipment is proposed to be carried out on the basis of a random risk process that describes the state of the insurance fund. This approach allowed us to introduce the concept of resource-cost risk, which allows to quantify the effectiveness of repair work in terms of their financial support. For his research, a simulation program based on the event approach was created. Experiments using a simulation program led to the conclusion that, from the point of view of reducing the resource-cost risk, the excess of income over expenditure must be done at the expense of the initial annual value of the insurance fund. Some conclusions and statements are logically obvious, but the analysis by the method of simulation modeling allows one to substantiate them quantitatively.
\end{abstract}

Keywords: robotic equipment, random risk process, resource-cost risk, simulation modeling.

Krakovsky Y. M. - Dr. Tech. Sci., Professor, Professor of Department of Information Systems and Information Security, Irkutsk State Transport University. E-mail: 79149267772@yandex.ru

Hoang N. A. - PhD student of the Department of Informatics and Mathematical Modeling of Irkutsk State Agricultural University named after A. A. Ezhevsky. E-mail: hoangngocanhmta@gmail.com 